\title{
Effects of Psycho-education Interventions on Perceived Childbirth Fear and Anxiety by Pregnant Women: A Systematic Review and Meta-analysis
}

\author{
Masomeh Adeli Gargari', Khalil Esmailpour ${ }^{2}$, Mojgan Mirghafourvand ${ }^{3}$, Roghaiyeh Nourizadeh ${ }^{4}$, \\ Esmat Mehrabi ${ }^{* *}$
}

\begin{abstract}
Objectives: Phobia of natural childbirth is one of the most important and influencing factors leading women to request cesarean sections. The present study aimed to prepare a systematic review and meta-analysis to investigate the type of interventions related to fear of childbirth (FOC).

Materials and Methods: The data collection was based on an extensive search of articles related to clinical trials (1990-2019) posted on Google Scholar, Cochran, SID, Magiran, Web of Science, Scopus, and PubMed electronic databases. In general, 109 abstracts were selected after eliminating similar publications. As a result of the qualitative evaluations of these articles, 11 studies were kept for investigation.

Results: The studies were categorized into three groups including prenatal education with childbirth counseling, yoga courses, and psychoeducational-based interventions. Based on the meta-analysis, psychoeducational interventions have a significant effect on childbirth fears and anxiety in comparison with the control group (mean difference: $0.85,95 \% \mathrm{Cl}:-1.20-0.45, P<0.0001, \mathrm{I}^{2}=82 \%$ ). Conclusions: Psychologically-based interventions, can creatively play an essential role in reducing the FOC during pregnancy and even childbirth. It can also create a pleasant and traumatic-free experience which is essential for encouraging women to have a natural childbirth.

Keywords: Phobia of childbirth (Tokophobia), Counseling, Psychodrama, Systematic review
\end{abstract}

\section{Introduction}

Fear of pain is a natural phenomenon and plays a vital role in human health. Fear arises when one considers pain-related stimuli as a threat (1). The birth process can be a potentially threatening phenomenon for the mother because it is associated with pain and the possibility of harm to both the mother and her fetus. Severe fear of childbirth (FOC) can be manifested as daily anxieties, nightmares, and physical complaints, causing maternal demand for a tendency toward cesarean sections $(2,3)$. The concept of FOC is a highly negative feeling about childbirth that can manifest itself as anxiety and fear during childbirth and tocophobia, which is a pathological fear and leads to the avoidance of childbirth. Most pregnant women express their FOC (4). FOC ranges from severe to a rational FOC, and most women, especially primiparous women, are unfamiliar with the process of childbirth. However, fear is most often experienced reasonably and is naturally controlled during pregnancy and childbirth (5). The results of previous studies show that one out of every five pregnant women has a FOC, and between 6\% and 36\% of pregnant women experience severe and disabling fear $(3,6,7)$.

Generally, the FOC is two-folds, including the primary fear that occurs in primiparous women and the secondary fear, which is caused by traumatic childbirth or previous problems $(8,9)$. Studies in this area demonstrate that FOC is subordinate to and part of anxiety and is caused by three factors. First, one's interpretation of the situation as an annoying issue can be the cause of tokophobia. The second cause might be replacing encounters (i.e., watching someone else giving birth). The last cause, as classified by some studies, is getting inaccurate information about childbirth, including hearing horror stories about childbirth and internalizing negative stories of other women $(10,11)$. There are also some biological factors that can induce childbirth fear, the fear of pregnancy, fear of physical harm, or fear of child or one's death. The psychological causes of FOC are personality traits, history of traumatic life events or traumatic experiences related to pregnancy, and previous childbirth or previous difficulty, and feelings of hopelessness or anxiety about maternity. 
The social causes of tokophobia can be due to problems with a partner, lack of social support, low socioeconomic status, and hearing horror stories about Liber from family, friends, acquaintances, and media (13-15).

It should be noticed that FOC for any reason can have serious side effects on the mother and the fetus. Pregnant women with anxiety represent the signs of abnormal patterns of the fetal heart rate, anxiety, and eating disorders, low Apgar score, increased mortality at birth, low birth weight because of increased uterine artery resistance (16), negative mood, anxiety, and depression (17). The other signs were aggression, a feeling of emergency, difficulty in achieving relaxation, emotional reactions in stressful situations, fear of losing control, and inappropriate and illogical behaviors $(7,18,19)$. Women who experience high levels of fear during pregnancy in addition to emotional imbalances and depression after childbirth (20). In addition, severe FOC and worrying about the labor process lead to the release of catecholamines, cortisol, and epinephrine. In severe cases, this leads to inadequate uterine contractions, and ultimately, inappropriate progress of the Liber. Additionally, psychological tensions, worrying and fear of delivery pain, lack of confidence in the delivery room staff, and stress from entering the unknown room (i.e., the delivery room) can lead to increased Liber time. Moreover, they result in increased pain caused by catecholamines, cortisol, and epinephrine released to overcome such tensions (21-24). Consequently, all these actions would increase pain and prolong the delivery process $(22,24,25)$.

Some studies have shown that a group of women can overcome fear while others need counselors' professional advice and assistance to manage their pregnancy and delivery without psychological trauma (26-28). Thus, educating women by midwives, gynecologists, and psychologists, if necessary, can be effective in preventing the effects of FOC $(26,27,29)$. Women's reaction to FOC includes a range of psycho-physiological (e.g., increased muscle activity), behavioral (e.g., escaping and avoidance), and cognitive (e.g., catastrophic thoughts) responses (30).

Therefore, the first important step in formulating, presenting, and applying guidelines and strategies for childbirth fear is to know what interventions might be effective in reducing childbirth fear. Different studies have investigated the effects of different interventions and strategies on reducing the FOC.

\section{Materials and Methods}

Extensive search and review of articles dealing with childbirth fear and the impact of different interventions were performed by searching English words such as intervention, consultation, fear of birth, and birth anxiety in published articles in Google Scholar, Cochran, SID, Magiran, Web of Science, PubMed, and Embase databases. The selection of corpus was also conducted by searching the keywords of delivery, phobia, anxiety, and educational interventions and counseling among Persian articles. It is important to note that all articles were published from 1990 to 2019. Further search was performed with the same strategy among dissertations, books, and available unpublished articles.

Resources were managed using Endnote software. For selecting the corpus, the title and abstract of related articles were studied, and qualified and related articles were opted for. Moreover, an expert in the field was consulted for selecting doubtful articles. Our search strategy consisted of reviewing the published Persian and English articles with distinct designs such as clinical trials and experimental or quasi-empirical studies. Studies, whose outcome was FOC, were systematically reviewed in this research, and studies examining anxiety of childbirth were also included in the study. Moreover, studies aiming at investigating educational and counseling interventions were considered as the research corpus. In general, all studies somehow examining effective theories, models, and interventions in the reduction of childbirth fear were selected for the study purpose. The selected articles were scrutinized without any considerations of cultural and social differences. The Cochrane tool, which is a specialized means of a systematic review of clinical trial articles, was implemented to evaluate the quality of trial studies. This tool examines the studies in six steps. The characteristics and the judgment methods of the 11 articles are indicated in Table 1.

\section{Results}

Nine out of 11 studies were randomized controlled trials, and one case was quasi-experimental research (Figure 1). The studies in this systematic review were categorized into four groups based on the type of interventions. In fact, the intervention of the two studies was prenatal education. The other two studies were of delivery counseling type. Moreover, two studies selected yoga and relaxation in pregnancy as their inventions. In addition, five studies were based on psychotherapy (cognitive-behavioral and group psychotherapy). Based on the meta-analysis, psychoeducational interventions have a significant effect on childbirth fear and anxiety in comparison with the control group (mean difference: 0.85, 95\% CI: -1.200.45, $\left.P<0.0001, I^{2}=82 \%\right)$. Notably, the FOC was a common factor that motivated all these studies. The studies are described as follows:

\section{Birth Counseling}

Ryding et al conducted a study entitled the "evaluation of midwives' counseling of pregnant women in FOC" during pregnancy. The study was conducted at the Helsingborg Hospital by a team of eight midwives who consulted women with FOC. The counseling procedure lasted for one to four session(s), mainly an average of four sessions, from 6 to 37 weeks of gestation and contained at least one postpartum session. It is noteworthy that postpartum 


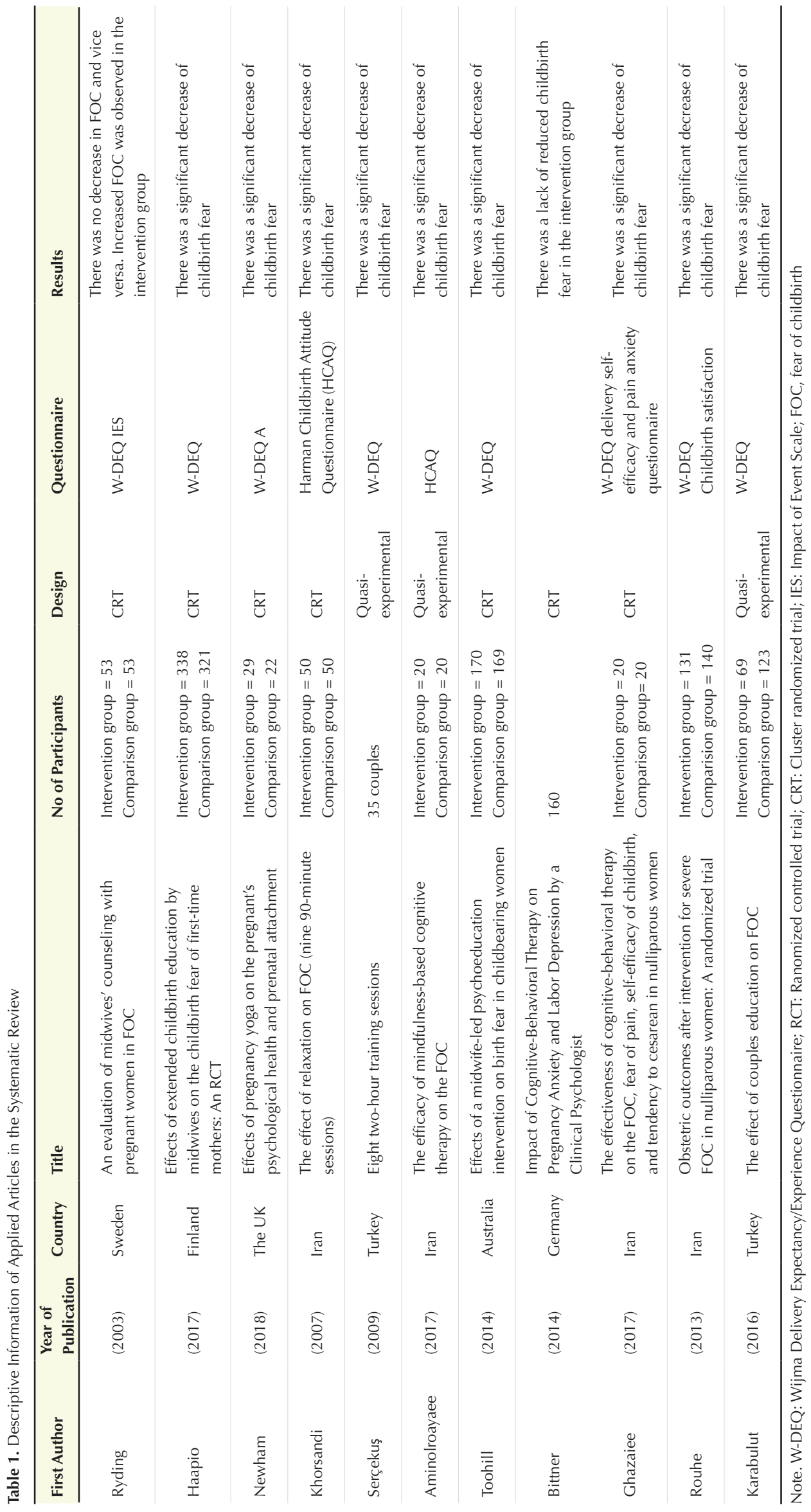




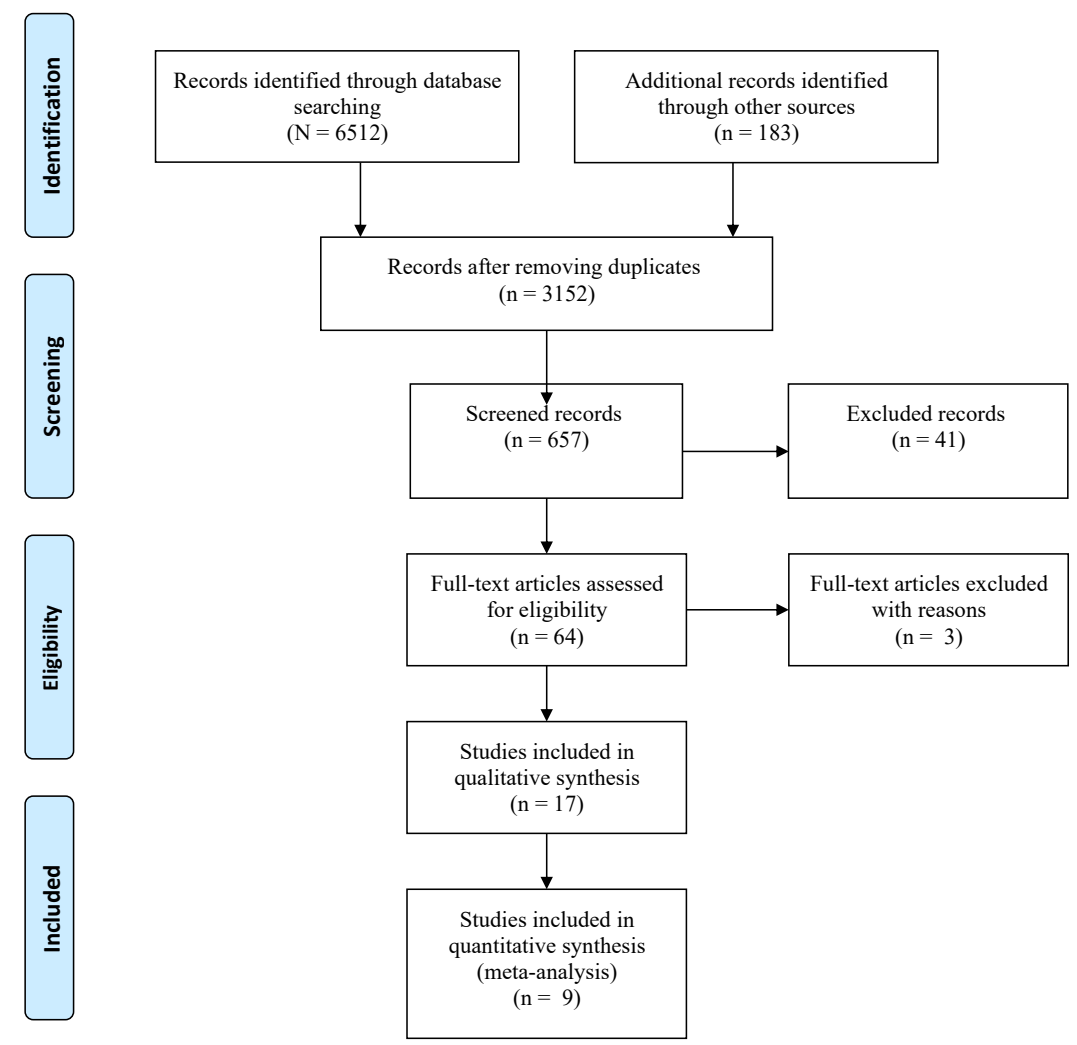

Figure 1. PRISMA Flowchart of the Study.

counseling lasted for three sessions in some cases. Of all consulted women, 62 cases met the inclusion criteria, 53 of whom participated in the study. Both intervention and control groups completed the Wijma Delivery Expectancy/Experience Questionnaire (W-DEQ) and the Impact of Event Scale. The results of this study showed that there was a significant difference between intervention and comparison groups after counseling. The symptoms of fear of treatment did not diminish, and even the symptoms of fear increased in comparison to the control group. $(\mathrm{P}=0.085)(2)$.

Yoga and Relaxation Courses

Newham et al studied the effects of pregnancy yoga on pregnant's psychological health and prenatal attachment in the UK $(n=59)$. The women in the intervention group were randomly assigned to 8 weeks of prenatal yoga compared to standard care. The sessions had a variety of topics to emphasize how yoga is used to promote maternal well-being. For example, the first to third sessions focused on reducing the common pain and pain caused by yoga, sessions 6 and 7 included training on positions to improve flexibility and breathing techniques during labor, and session 8 focused on pelvic floor exercises. Women in the control group typically received ordinary prenatal care. After the intervention, FOC decreased in the intervention group compared to the control group (31). In addition, in a clinical trial, Khorsandi et al examined the effects of relaxation on FOC among 100 primiparous women who referred to Milad hospital in Tehran for prenatal care. Subjects were divided into intervention and control groups each containing 50 cases. The control group received only routine care while the intervention group was divided into five groups containing ten subjects, each group receiving nine sessions of 90-minute training from the second trimester. The results of this study revealed that relaxation training was significantly $(P<0.001)$ effective in reducing childbirth fear while increasing natural childbirth (32).

Prenatal Education

Haapio et al (33) selected 659 Finnish pregnant women at first birth to provide extensive midwifery (intervention) or standard prenatal (voluntary childbirth education class at the health clinic and a brief visit to the obstetrics ward) education. The intervention included standard prenatal care, along with a pamphlet with basic information on pregnancy and childbirth and a two-hour training course on labor. The women attended the meeting before the 34th week of pregnancy. The session took place in the delivery room and included several exercises to learn different pain relief techniques. Birth fears of women in the intervention group were significantly lower compared to the control group (OR: 0.58; 95\% CI: 0.38-0.88).

Likewise, Serçekuş and Başkale (34) evaluated 35 Turkish pregnant couples attending eight two-hour prenatal training sessions. These sessions included 
topics such as physiological and psychological changes during pregnancy on how to cope with these changes, feelings of childbirth, and a discussion on how to manage childbirth fear, delivery and labor pain mechanisms, breathing and relaxation techniques, labor pain situations, breastfeeding, infant care, and the like. In addition to receiving daycare, the couples of the intervention group participated in delivery training. Women or couples in the control group received only prenatal care. Participation in childbirth education classes was associated with reduced FOC in women so that the mean scores of the pregnancy and childbirth of the experimental group in the W-DEQ questionnaire decreased from 60.7 points at baseline to 37.9 after treatment. The difference between the scores of pregnancy and childbirth questionnaires in the intervention and control groups after treatment was significant (37.9 vs. $59.9 \%)$.

Psychotherapy: Cognitive-Behavioral and Group Psychotherapy

In a clinical trial, Aminolroayaee and Aghaei (35) investigated the efficacy of mindfulness-based cognitive therapy on the FOC among 40 primiparous women who referred to Amin hospital in Isfahan in spring 2017. Participants were divided into intervention $(n=40)$ and control groups $(n=40)$. Childbirth Attitude Questionnaire (CAQ) was used to measure the FOC. The control group received only routine care whereas the intervention group received eight 60 minute sessions of a mindfulness-based cognitive therapy group. The post-test was performed after eight sessions in both groups and one month after the last session. The effectiveness of the viability of childbirth fear was again evaluated in both groups. The results of this study demonstrated that mindfulnessbased cognitive therapy reduced fear of natural childbirth and sustained its reduced level $(P<0.05)$. In a clinical controlled trial, Toohill et al examined the effects of a midwife-led psychoeducation intervention on birth fear in childbearing women in Australia from May 2012 to June 2013. Participants were divided into intervention $(n=170)$ and control $(n=169)$ groups both receiving handbooks to help them make decisions about the type of delivery. The intervention group received telephone counseling from midwives from weeks 24 to 34 , and the control group received routine care. The primary outcome was a decrease in FOC from the second trimester to week 36 of gestation. Moreover, the secondary outcome was a promotion of childbirth self-efficacy and a decrease in depression symptoms and reduced decision conflicts. There was a statistically significant difference between the intervention and control groups in fear of delivery $(P<0.001)$ and self-efficacy $(P=0.002)$ after the intervention (36). In Germany, Bittner et al randomly selected 160 women and assigned them to 8 weeks of psychotherapy or cognitive-behavioral therapy by a clinical psychologist in addition to standard prenatal care. Women participating in the study reported prenatal, anxiety, or depression in standardized clinical interviews. Women with severe depression, anxiety, or other acute psychological problems were excluded from the study and referred for treatment. In the intervention group, 4-6 pregnant women participated in an 8-week course to be trained in cognitive-behavioral therapy, advanced muscle relaxation, dealing with stress, problem-solving techniques, prevention and treatment of pregnancy anxiety and depression during pregnancy and be educated in all required issues for the postpartum period. The women in the control group received only standard prenatal care. Based on the results of the study, there was no significant difference between the socio-demographic characteristics of the women in the treatment and control groups. As a result of the cognitive-behavioral therapy intervention, no significant reduction was found in FOC (37). In a clinical controlled trial, Ghazaie et al (38) investigated the efficacy of cognitive-behavioral therapy on the fear of delivery, fear of pain, self-efficacy, and the cesarean section on 25 primiparous women in Mashhad. Subjects were assigned to intervention and control groups, and nine sessions of cognitive-behavioral therapy were administered by the interventionist psychologist on the intervention group. W-DEQ, self-efficacy, and pain anxiety questionnaires were used as pre- and post-test tools, respectively. Pain and tendency to cesarean section significantly decreased in the intervention group compared to the control group $(P<0.05)$. In a randomized clinical trial, Rouhe et al studied the impact of group-based psychotherapy on coping with the FOC. The intervention and control groups each consisted of 135 individuals. The intervention method, which was provided by four different psychologists with specialized therapeutic skills, was applied to treat women with severe FOC through a psychiatric counseling treatment. The counselor needed to work with groups involving six women. The group counseling started from the 16th week and lasted for six sessions during pregnancy and one session after 6-8 weeks of childbirth. The content of the sessions included the pleasant focus of mind (visualization of flower bud opening), information on FOC and delivery stages in the hospital, parental and fetal preparation, and postpartum evaluation and counseling on physical and mental problems. The W-DEQ (Version A) and W-DEQ (Version B) delivery questionnaires were used to assess fear. Additionally, the satisfaction questionnaire was completed in the second trimester of pregnancy and at the end of the third trimester, and three months after delivery. Their results showed that the score of FOC significantly decreased in the intervention group after counseling (39). Based on the meta-analysis in the present study (Figure 2), psychoeducational interventions have a significant effect on childbirth fears and anxiety in comparison with the control group (mean difference: 0.85, 95\% CI: $\left.-1.20-0.45, P<0.0001, \mathrm{I}^{2}=82 \%\right)$. 


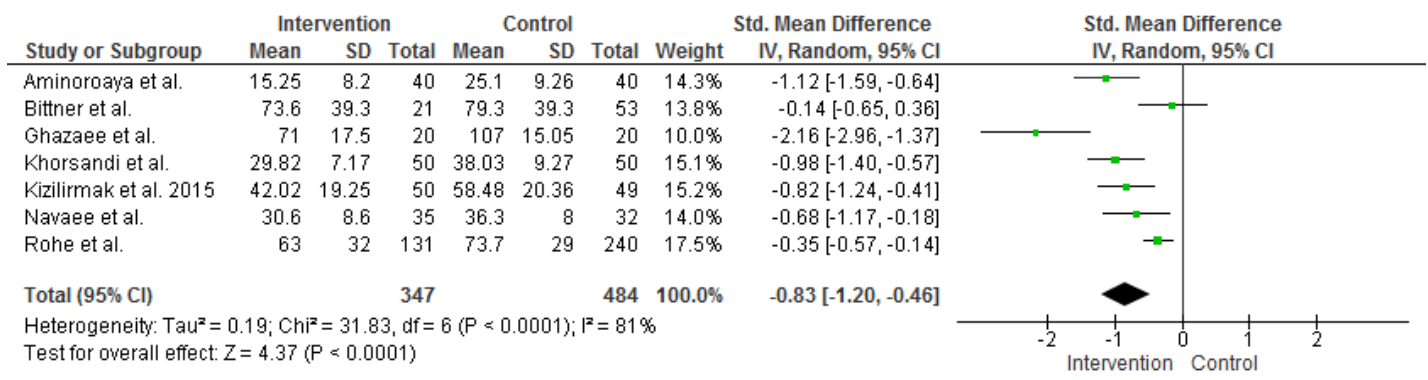

Figure 2. Effects of Psycho-education Interventions on Perceived Childbirth Fear and Anxiety by Pregnant Women.

\section{Discussion}

Overall, 10 (66\%) out of 15 studies had sufficient quality for the study and thus were systematically reviewed in this research. Based on the findings of studies by Ryding et al evaluating midwives' counseling of pregnant women in FOC (30) and Bittner et al scrutinizing cognitivebehavioral therapy intervention (2), significant changes were found in FOC after the intervention. The results of studies including the mindfulness-based cognitive therapy intervention (35), cognitive-behavioral therapy intervention (38), relaxation intervention on FOC, and group-based psychological intervention (39) indicated that short-term personal psychotherapy interventions can be effective for FOC and pregnancy-specific anxiety. Interventions that were effective for pregnant women with varying levels of FOC included hospital-related education (33), prenatal yoga, and prenatal training (34).

However, two studies of cognitive-behavioral therapy on childbirth fear by Bittner et al and Ghazaie et al reported contradictory results. Bittner et al (2) found no decrease in childbirth fear. Conversely, Ghazaie et al (38) reported a significant decrease in this regard. Nonetheless, further studies are required in this area. Another study providing midwifery-related standard training on pregnancy and childbirth reported a significant reduction in the FOC (33).

In addition to the above-mentioned research, Karabekir presented articles in 2016 and 2017 providing a model for the delivery team and introducing the psychodrama approach and its impact on the fear of delivery, thus, making the delivery process more pleasant for the mother and family. In their essay, they described the psychodrama and how it was used by a psychologist during prenatal education and the delivery process, focusing on psychological issues during childbirth. They also pointed out the impact of creative use of psychodrama on the delivery process and the maternal experience and even emphasized eliminating previous unpleasant childbirth experiences. Their delivery team model points to the need for the attendance of a psychodramaist in addition to the presence of a gynecologist and a midwife. Their study provides more tangible cases and researcher experiences which supply creative use of psychodrama in having a satisfactory delivery. Furthermore, they mentioned the delivery environment as one of the most important factors in the delivery process and emphasized the necessity of a relaxed environment in which mothers can deliver their fetuses $(40,41)$.

To sum up, the results of a systematic review of these studies indicated the effectiveness of the psychiatric interventions of pregnant women during pregnancy and delivery. Cognitive-behavioral interventions, which were used in the selected studies as the corpus, mainly considered FOC as a central phenomenon. They also focused on the positive impact of the interventions of psychological types on women's weaknesses regarding natural childbirth. In fact, women were reported to reduce FOC $(2,38)$. It is important to note that the use of psychological approaches, especially the psychodrama method, in a creative way during pregnancy and even childbirth, can play an important role in reducing the FOC and creating a pleasant and free experience of delivery. Presumably, a mental-trauma free environment is necessary and an emergency for encouraging women to have a natural childbirth.

\section{Suggestions for Future Research}

It is recommended that further interventional studies be conducted regarding childbirth fear of both couples simultaneously. It was noted that the presence of the husband in counseling sessions played a trivial role in the available studies. Further, there is a need to examine different models in the delivery team setting. Further studies are also required on psychodrama and its impact on childbirth and childbirth experience.

A key feature in all future studies should be the use of valid measures of FOC. In the above-mentioned systematic study, it was found that the W-DEQ was excessively used in assessing FOC during pregnancy. Although this questionnaire has good psychometric abilities (42), it suffers from a number of features. First, W-DEQ examines a wide range of women's perceptions and feelings about labor pain thus it is not limited to assessing childbirth fears.

More importantly, several aspects of childbirth fear (e.g., perception of social embarrassment, the pressure to take or avoid pain medications, maternal safety, changes in the body and sexual function, and fear of medical 
interventions) have not been addressed in this criterion (3).

\section{Conclusions}

The findings of this study can provide useful information for delivery teams regarding the use of a variety of creative and counseling-based methods during pregnancy and delivery to improve the delivery process. The findings of this review study may identify the origins of FOC (including previous psychological problems during pregnancy and childbirth) to help women develop strategies to cope with fear. Finally, the strategies can include prenatal education and training, including being in the delivery room, advanced muscle relaxation, and other techniques to combat stress and fear of pregnancy.

\section{Authors' Contribution}

The first and correspond authors performed an initial search of databases and were major contributors in writing the manuscript. Second and fifth authors reviewed studies to investigate eligibility criteria. The third author regulated methods and performed a meta-analysis. All authors read and approved the final manuscript.

\section{Conflict of Interests}

Authors declare that they have no conflict of interests.

\section{Ethical Issues}

Not applicable.

\section{Financial Support}

This research was supported by Tabriz University of Medical Sciences financially as the part of MSC thesis at faculty of Nursing and Midwifery at Tabriz University of Medical Sciences.

\section{References}

1. Leeuw M, Goossens ME, Linton SJ, Crombez G, Boersma K, Vlaeyen JW. The fear-avoidance model of musculoskeletal pain: current state of scientific evidence. J Behav Med. 2007;30(1):7794. doi:10.1007/s10865-006-9085-0

2. Nieminen K, Stephansson O, Ryding EL. Women's fear of childbirth and preference for cesarean section--a cross-sectional study at various stages of pregnancy in Sweden. Acta Obstet Gynecol Scand. 2009;88(7):807-813. doi:10.1080/00016340902998436

3. Rouhe H, Salmela-Aro K, Halmesmäki E, Saisto T. Fear of childbirth according to parity, gestational age, and obstetric history. BJOG. 2009;116(1):67-73. doi:10.1111/j.1471-0528.2008.02002.x

4. Gao LL, Liu XJ, Fu BL, Xie W. Predictors of childbirth fear among pregnant Chinese women: a cross-sectional questionnaire survey. Midwifery. 2015;31(9):865-870. doi:10.1016/j.midw.2015.05.003

5. Salomonsson B, Wijma K, Alehagen S. Swedish midwives' perceptions of fear of childbirth. Midwifery. 2010;26(3):327-337. doi:10.1016/j.midw.2008.07.003

6. Haines H, Pallant JF, Karlström A, Hildingsson I. Cross-cultural comparison of levels of childbirth-related fear in an Australian and Swedish sample. Midwifery. 2011;27(4):560-567. doi:10.1016/j. midw.2010.05.004

7. Alipour Z, Lamyian M, Hajizadeh E, Agular Vafaei M. The association between antenatal anxiety and fear of childbirth in nulliparous women: a prospective study. Iran J Nurs Midwifery Res. 2011;16(2):169-173.

8. Alehagen S, Wijma K, Wijma B. Fear during labor. Acta Obstet Gynecol Scand. 2001;80(4):315-320. doi:10.1034/j.16000412.2001.080004315.x

9. Ryding EL, Wijma B, Wijma K, Rydhström H. Fear of childbirth during pregnancy may increase the risk of emergency cesarean section. Acta Obstet Gynecol Scand. 1998;77(5):542-547. doi:10.1034/j.1600-0412.1998.770512.x

10. Rondung E, Thomtén J, Sundin Ö. Psychological perspectives on fear of childbirth. J Anxiety Disord. 2016;44:80-91. doi:10.1016/j. janxdis.2016.10.007

11. Fenwick J, Toohill J, Creedy DK, Smith J, Gamble J. Sources, responses and moderators of childbirth fear in Australian women: a qualitative investigation. Midwifery. 2015;31(1):239-246. doi:10.1016/j.midw.2014.09.003

12. Engel GL. The need for a new medical model: a challenge for biomedicine. Fam Syst Med. 1992;10(3):317-331. doi:10.1037/ h0089260

13. Hofberg K, Ward MR. Fear of childbirth, tocophobia, and mental health in mothers: the obstetric-psychiatric interface. Clin Obstet Gynecol. 2004;47(3):527-534. doi:10.1097/01. grf.0000132527.62504.ca

14. Saisto T, Salmela-Aro K, Nurmi JE, Halmesmäki E. Psychosocial characteristics of women and their partners fearing vaginal childbirth. BJOG. 2001;108(5):492-498. doi:10.1111/j.14710528.2001.00122.x

15. Zar M, Wijma K, Wijma B. Pre- and postpartum fear of childbirth in nulliparous and parous women. Scand J Behav Ther. 2001;30(2):75-84. doi:10.1080/02845710121310

16. Räisänen $S$, Lehto $S M$, Nielsen HS, Gissler M, Kramer MR, Heinonen S. Fear of childbirth in nulliparous and multiparous women: a population-based analysis of all singleton births in Finland in 1997-2010. BJOG. 2014;121(8):965-970. doi:10.1111/1471-0528.12599

17. Hall WA, Hauck YL, Carty EM, Hutton EK, Fenwick J, Stoll K. Childbirth fear, anxiety, fatigue, and sleep deprivation in pregnant women. J Obstet Gynecol Neonatal Nurs. 2009;38(5):567-576. doi:10.1111/j.1552-6909.2009.01054.x

18. Nasiry F, Sharifi S. Relationship between fear of childbirth and personality type in pregnant women. Iran J Obstet Gynecol Infertil. 2013;16(66):18-25. doi:10.22038/ijogi.2013.1901

19. Khorsandi M, Vakilian K, Nasirzadeh Masooleh M. Investigating different factors of fear in normal delivery among pregnant women, in Arak-a cross sectional study. J Fasa Univ Med Sci. 2014;4(2):161-167. [Persian].

20. Akhlaghi F, Mokhber N, Shakeri MT, Shamsa F. Relation between depression, anxiety, self-esteem, marital satisfaction, demographical factor and maternal complications with fear of childbirth in nulliparous women. J Fasa Univ Med Sci. 2012;14(2):122-131. [Persian].

21. Pirdel M, Pirdel L. Perceived environmental stressors and pain perception during labor among primiparous and multiparous women. J Reprod Infertil. 2009;10(3):217-223.

22. Keshavarz M, Shariati M, Jahdi F. Effects of complementary therapies on pain and labor outcomes in nuliparous women referred to delivery unit in Fatemiyeh hospital in Shahrood city (2003-2005). Medical Science Journal of Islamic Azad UnivesityTehran Medical Branch. 2008;18(4):245-250. [Persian].

23. Nichols F. Philosophy and roles. In: Nichols FH, Humenick SS, eds. Childbirth Education: Practice, Research, and Theory. 2nd ed. Philadelphia: Saunders; 2000:3-17.

24. Murray SS, McKinney ES, Gorrie TM. Clinical Companion for Foundations of Maternal-Newborn Nursing. 4th ed. Philadelphia: Saunders; 2006:512.

25. Nichols FH, Humenick SS. Childbirth Education: Practice, Research and Theory. 2nd ed. U.S.A: WB Saunders Company; 2000:731.

26. Saisto T, Salmela-Aro K, Nurmi JE, Könönen T, Halmesmäki E. A randomized controlled trial of intervention in fear of childbirth. Obstet Gynecol. 2001;98(5 Pt 1):820-826. doi:10.1016/s00297844(01)01552-6

27. Nerum H, Halvorsen L, Sørlie T, Oian P. Maternal request for cesarean section due to fear of birth: can it be changed through crisis-oriented counseling? Birth. 2006;33(3):221-228. 
doi:10.1111/j.1523-536X.2006.00107.x

28. Saisto T, Toivanen R, Salmela-Aro K, Halmesmäki E. Therapeutic group psychoeducation and relaxation in treating fear of childbirth. Acta Obstet Gynecol Scand. 2006;85(11):1315-1319. doi:10.1080/00016340600756920

29. Ryding EL. Investigation of 33 women who demanded a cesarean section for personal reasons. Acta Obstet Gynecol Scand. 1993;72(4):280-285. doi:10.3109/00016349309068038

30. Ryding EL, Persson A, Onell C, Kvist L. An evaluation of midwives' counseling of pregnant women in fear of childbirth. Acta Obstet Gynecol Scand. 2003;82(1):10-17. doi:10.1034/j.16000412.2003.820102.x

31. Newham JJ, Wittkowski A, Hurley J, Aplin JD, Westwood M. Effects of antenatal yoga on maternal anxiety and depression: a randomized controlled trial. Depress Anxiety. 2014;31(8):631640. doi:10.1002/da.22268

32. Khorsandi M, Ghofranipour F, Heydarnia A, et al. The effect of childbirth preparation classes on childbirth fear and normal delivery among primiparous women. J Arak Univ Med Sci. 2008;11(3):29-36. [Persian].

33. Haapio S, Kaunonen M, Arffman M, Åstedt-Kurki P. Effects of extended childbirth education by midwives on the childbirth fear of first-time mothers: an RCT. Scand J Caring Sci. 2017;31(2):293301. doi:10.1111/scs.12346

34. Serçekuş $P$, Başkale H. Effects of antenatal education on fear of childbirth, maternal self-efficacy and parental attachment. Midwifery. 2016;34:166-172. doi:10.1016/j.midw.2015.11.016

35. Aminolroayaee M, Aghaei A. The effectiveness of cognitive therapy based on mindfulness on fear of delivery in primiparous women. Knowl Res Appl Psychol. 2019;19(4):55-65. [Persian].
36. Toohill J, Fenwick J, Gamble J, et al. A randomized controlled trial of a psycho-education intervention by midwives in reducing childbirth fear in pregnant women. Birth. 2014;41(4):384-394. doi:10.1111/birt.12136

37. Bittner A, Peukert J, Zimmermann C, et al. Early intervention in pregnant women with elevated anxiety and depressive symptoms: efficacy of a cognitive-behavioral group program. J Perinat Neonatal Nurs. 2014;28(3):185-195. doi:10.1097/jpn.0000000000000027

38. Ghazaie M, Davoodi I, Neysi A, Mehrabizadeh Honarmand M, Bassak Nejad S. The effectiveness of cognitive-behavioral therapy on fear of childbirth, fear of pain, self-efficacy of childbirth and tendency to caesarean in nulliparous women [Persian]. Iran J Obstet Gynecol Infertil. 2016;19(31):1-12. doi:10.22038/ ijogi.2016.7989

39. Rouhe H, Salmela-Aro K, Toivanen R, Tokola M, Halmesmäki E, Saisto T. Obstetric outcome after intervention for severe fear of childbirth in nulliparous women - randomised trial. BJOG. 2013;120(1):75-84. doi:10.1111/1471-0528.12011

40. Karabekir N. Using Psychodrama in Childbirth Education and Birth Psychotherapy: Birth with No Regret. J Prenat Perinat Psychol Health. 2016;30(3):208-217.

41. Karabekir N. Birth psychodrama: what is the role of the birth psychodramatist/psychotherapist in the labour ward? Zeitschrift für Psychodrama und Soziometrie. 2017;16(1):5-14. doi:10.1007/ s11620-017-0374-0

42. Wijma K, Wijma B, Zar M. Psychometric aspects of the W-DEQ; a new questionnaire for the measurement of fear of childbirth. J Psychosom Obstet Gynaecol. 1998;19(2):84-97. doi:10.3109/01674829809048501

(C) 2021 The Author(s); This is an open-access article distributed under the terms of the Creative Commons Attribution License (http:// creativecommons.org/licenses/by/4.0), which permits unrestricted use, distribution, and reproduction in any medium, provided the original work is properly cited. 